\title{
La conscience disciplinaire
}

Présentation d'un concept

Yves Reuter

\section{OpenEdition}

\section{Journals}

Édition électronique

URL : https://journals.openedition.org/educationdidactique/175

DOI : 10.4000/educationdidactique. 175

ISBN : 978-2-7535-1615-1

ISSN : 2111-4838

\section{Éditeur}

Presses universitaires de Rennes

\section{Édition imprimée}

Date de publication : 1 septembre 2007

Pagination : 55-71

ISBN : 978-2-7535-0534-6

ISSN : 1956-3485

\section{Référence électronique}

Yves Reuter, "La conscience disciplinaire », Éducation et didactique [En ligne], 1-2 | septembre 2007, mis en ligne le 01 septembre 2009, consulté le 15 février 2023. URL : http://journals.openedition.org/ educationdidactique/175; DOI : https://doi.org/10.4000/educationdidactique.175

\section{c) ()ㅇㄱ}

Creative Commons - Attribution - Pas d'Utilisation Commerciale - Pas de Modification 4.0 International - CC BY-NC-ND 4.0

https://creativecommons.org/licenses/by-nc-nd/4.0/ 


\title{
LA CONSCIENCE DISCIPLINAIRE présentation d'un concept
}

\author{
Yves Reuter \\ Université Charles de Gaulle - Lille 3 \\ Equipe THEODILE (E.A. 1764)
}

\begin{abstract}
Résumé : Cet article soumet à la discussion le concept de conscience disciplinaire qui désigne la manière dont les acteurs sociaux et plus particulièrement les élèves (re)construisent les disciplines scolaires. Après en avoir retracé la genèse au travers des questions qui ont suscité son élaboration et les travaux antérieurs qui manifestaient sa nécessité, l'auteur expose les premiers résultats que sa mise en œuvre a permis d'obtenir (reconnaissance, repérage, finalités des disciplines...). Puis il expose les problèmes soulevés par ce concept : choix définitionnels, méthodes d'investigation... Il conclut en montrant quelques-uns des principaux intérêts de son usage en didactique(s) : autonomisation de l'appareil conceptuel, instrument de comparaison entre disciplines, spécification de pistes de recherche autour de l'échec, de la relation entre écoles et familles ou encore de l'importance de l'ordre scriptural.

Mots-clés : conscience disciplinaire ; discipline scolaire ; représentation ; clarté cognitive ; didactique comparée ; échec scolaire.
\end{abstract}

Yves Reuter

Je me propose ici de soumettre à la discussion un concept, celui de conscience disciplinaire, dont j'ai effectué une première présentation dans un article relativement récent (Reuter, 2003 ${ }^{1}$ ), et qui fait l'objet de multiples travaux, notamment au travers de mémoires de Master ${ }^{2}$ au sein de notre laboratoire.

À cette fin, je commencerai par rappeler sa définition, certains éléments de sa genèse, les modalités de sa mise en œuvre méthodologique et quelquesuns des principaux résultats obtenus ainsi que les questions qui l'accompagnent. J'exposerai ensuite les problèmes qu'il soulève et les intérêts qu'il présente en m'attachant plus particulièrement à sa pertinence possible au sein du projet de connaissance des didactiques et de la didactique comparée.

\section{La conscience disciplinaire. Présentation d'un concept}

\section{Éléments pour une définition}

J'avais proposé de définir la conscience disciplinaire comme la manière dont les acteurs sociaux, et plus particulièrement les acteurs scolaires, (re) construisent les disciplines scolaires. Cette définition, succincte, nécessite immédiatement deux précisions. En premier lieu, si nous nous sommes essentiellement intéressés jusqu'à présent dans nos recherches aux élèves, il nous parait tout aussi important d'étudier la conscience disciplinaire d'autres acteurs tels les maîtres, les parents d'élèves ${ }^{3}$, les formateurs, les inspecteurs... voire les chercheurs. En second lieu, j'insiste sur le fait que deux aspects au moins sont à considérer dans la mise en œuvre de ce concept. les formes de ces (re) constructions (les représentations des disciplines pouvant être structurées de manière différente par les apprenants, $c f$. 1.4.) et la plus ou moins grande clarté dont elles témoignent, sachant que la qualification même de clarté sera interrogée un peu plus loin dans cet article (cf. 2.1.).

\section{Éléments de genèse}

Il est toujours difficile de reconstituer l'histoire de l'émergence d'un concept, a fortiori si celle-ci est récente et si on s'y trouve fortement impliqué. Les quelques éléments qui suivent sont donc à considérer avec précaution.

Il me semble, en tous cas, que trois sources hétérogènes ont convergé pour permettre cette élaboration. celle de constats empiriques, celle de cheminements théoriques internes au champ des didactiques (voire propres aux recherches de notre équipe), celle d'analyses externes aux didactiques, mais présentant des intérêts certains pour celles-ci.

En ce qui concerne les constats empiriques, il m'a fallu finalement du temps pour prendre conscience de leur récurrence, de leur importance et de leurs connexions. Il s'agit notamment de déclarations d'élèves du primaire, pas nécessairement en difficulté qui, au travers d'entretiens formels ou informels, s'avéraient incapables d'indexer un cours ou un contrôle à une discipline. Le temps qu'il m'aura fallu pour prendre conscience de ces phénomènes et les intégrer comme objets possibles de questionnement en recherche me parait témoigner du poids des disciplines et des effets de naturalisation engendrés, rendant potentiellement aveugle aux problèmes de 
construction qu'ils peuvent poser aux apprenants. Ce poids de l'évidence disciplinaire m'a semblé confirmé par les résistances initiales de mes étudiants, souvent enseignants, à qui j'exposais cela. Ils étaient tellement convaincus que c'était clair pour les élèves tant ils posaient et répétaient de repères (horaires, supports, déclarations...) qu'il ne pouvait s'agir pour eux que de phénomènes marginaux. Bien souvent, ce n'est qu'après des investigations auprès de leurs propres élèves, qu'ils ont reconnu, à leur grand désarroi, le caractère ordinaire de ces «flottements ». Mais ceux-ci ne se limitent pas à l'école primaire. En effet, dans le secondaire, des entretiens, menés auprès de lycéens, manifestent des formes de construction de certaines disciplines pour le moins éloignées de leur structuration dans l'esprit de leurs concepteurs et de leurs enseignants. Le cas le plus frappant, mais aussi le plus récurrent, est sans doute celui du français comme discipline où il s'agit de « baratiner ».

Les questions engendrées par ces constats m'ont semblé pouvoir être mises en relation avec des réflexions ou des recherches attestées dans le champ des didactiques, bien que celles-ci n'aient pas, originellement, le même projet et qu'elles n'aient pas été, en conséquence, mises en réseau de manière explicite auparavant ${ }^{4}$. Je n'en prendrai ici que trois exemples. Le premier est issu des travaux de Jacques Colomb qui, proposant une relecture critique des concepts de contrat didactique et de contrat disciplinaire, avançait la notion «d'image» de la discipline (1993, p. 46) en prenant appui sur une recherche (Colomb, dir., 1987) explorant les ruptures et les continuités entre les enseignements en C.M. et en sixième. Le second exemple est celui de Nicole Lautier, dans son ouvrage, A la rencontre de l'histoire (1997), qui se donnait pour objet de recherche l'histoire apprise en confrontant les représentations de l'histoire et ses modes d'enseignement du côté des enseignants et les représentations de l'histoire et ses modalités d'appropriation du côté des élèves, cela en collège et en lycée. Le troisième exemple est associé aux travaux de l'équipe de didactique de Bordeaux autour de Jean-Paul Bernié. Ainsi, dans un article étudiant cohérence et positionnement énonciatif dans des copies d'élèves de C.M.2 en sciences, Martine Jaubert (2001) montrait bien comment toute une série de problèmes pouvaient être éclairés par la difficulté à entrer dans un discours disciplinaire (et non « ordinaire »).
Dans ces trois cas, il me semble que la notion de représentation de la discipline est fondamentale, sans que ce caractère stratégique lui soit réellement reconnu et qu'elle fasse, par voie de conséquence, l'objet d'une véritable construction et d'une réflexion critique sur sa pertinence dans un cadre peu ou prou didactique. M'intéresse en tout cas le fait que, dans nombre d'études aux projets de connaissance relativement différents, la notion apparaisse, même faiblement thématisée, comme un outil nécessaire.

Conjointement, la prise de conscience des problèmes soulevés par les constats empiriques m'a amené à relire un certain nombre de recherches menées au sein du laboratoire THEODILE à la lumière de ce questionnement.

Il s'agit, en premier lieu, des travaux menés sur la description dans les différentes disciplines scolaires ${ }^{5}$ (Cahiers Pédagogiques, 1999; Enjeux, 2000; Pratiques, 1998; Reuter, 2000; Reuter dir., 1998) qui ont permis, entre autres, de mettre au jour que, selon le cadre disciplinaire et les représentations qu'avaient les élèves de ce cadre disciplinaire, l'importance, le statut et les fonctions des descriptions n'étaient pas également identifiés et que, d'autre part, certains problèmes d'écriture pouvaient être interprétés soit en termes d'oblitération de modalités de fonctionnement de la description pourtant apprises par ailleurs (en français par exemple), soit en termes de transfert inapproprié de fonctionnements acquis dans un cadre disciplinaire au sein d'un autre cadre disciplinaire (les modalités de la description apprises en français transférées en mathématiques, par exemple).

Il s'agit, en second lieu, de travaux menés sur les représentations de l'écrit (lecture, écriture, conduites langagières...) dans différentes disciplines au collège (français, mathématiques, sciences, histoire et géographie). Dans cette perspective, nous avions pu montrer (Lahanier-Reuter et Reuter, 2002), entre autres, que la conscience de l'écrit variait selon le cadre disciplinaire et les représentations de ce cadre, avec tendanciellement une organisation des disciplines en deux catégories. l'une, représentée exemplairement par le français, au sein de laquelle l'écrit est considéré comme fondamental mais souvent réduit à du discours, des procédés purement formels, associés à des contenus « faibles »; l'autre, représentée exemplairement par les mathématiques, où l'écrit est secondaire ou, en tout cas, au service de contenus « forts ». Il convient encore de signaler que ces travaux étaient en étroite 
relation avec ceux que nous avons mené au sein de THEODILE sur les rapports à l'écriture et les images du scripteur en insistant sur la variabilité de ceuxci selon les espaces sociaux et scolaires (Delcambre et Reuter, 2002). On voit bien, ici encore, que si la conscience disciplinaire n'était pas explicitement thématisée, les résultats qui émergent de ces travaux mettent au jour des phénomènes qu'elle est susceptible d'éclairer.

C'est encore à la lumière de ces constats empiriques et d'un certain nombre de recherches évoquées que j'ai été amené à concevoir d'éventuelles connexions avec des travaux, notamment en psychologie de l'éducation, qui attiraient depuis un certain temps déjà l'attention sur des phénomènes liés à la clarté cognitive des apprenants quant aux apprentissages. Il s'agit principalement de ceux de Downing et Fijalkow (1984) et de ceux de Brossard (1994, $2002 \ldots$... . Dans le premier cas, les auteurs effectuant leurs recherches dans le domaine de l'apprentissage de la lecture, défendent la thèse selon laquelle une partie des difficultés d'un certain nombre d'élèves lors de l'entrée dans l'écrit pourrait être attribuable au fait que l'enseignement porte d'emblée sur des procédures techniques, alors que celles-ci ne font pas sens pour les apprenants, dans la mesure où ceux-ci ne disposent pas d'une clarté suffisante quant à l'écrit ou à la lecture ${ }^{6}$ (les principes du système écrit, les fonctions de la lecture, ses différences avec l'écriture...)... Dans le second cas, il s'agit de recherches portant sur l'articulation entre tâches et représentations de ces tâches et réussite ou échec scolaire. Dans cette perspective, Michel Brossard défend l'hypothèse, étayée de longue date sur de solides travaux empiriques, selon laquelle un des facteurs principaux de différenciation quant à la réussite ou l'échec scolaire tiendrait à l'inégale clarté des élèves face aux situations auxquelles ils sont confrontés (construction de leur relations, de leur sens, de leur finalité...). Par voie de conséquence, pour certains, la vie scolaire consiste en une juxtaposition de situations cloisonnées dont le sens s'épuise avec l'accomplissement de la réalisation de la consigne (ainsi, pour certains élèves, mettre en ordre des étiquettes s'effectuerait sans relation avec des situations antérieures ni avec des finalités telles qu'apprendre à lire ou à produire des phrases). Ces travaux s'effectuent sans doute dans une perspective différente de la nôtre mais leurs thèses et leurs résultats ne peuvent laisser indifférents les didacticiens et il m'a semblé qu'on pouvait établir des ponts avec nos questionnements dans la mesure où les prismes de l'organisation disciplinaire des enseignements et de la reconstruction du cadre disciplinaire par les apprenants sont susceptibles d'être envisagés comme des facteurs concourant à ou entravant la clarté des apprentissages telle qu'elle a été étudiée par ces auteurs.

Ainsi mise en perspective, la notion de conscience disciplinaire a pris sa place dans un double réseau de questionnements et de concepts. Le premier est lié à l'importance accordée au sein de notre laboratoire au concept de discipline (scolaire) et à son retravail à partir de deux postulats essentiels. il s'agit d'une construction (socio-scolaire); quatre espaces principaux concourent à cette construction. celui des prescriptions (via les textes officiels notamment), celui des recommandations (via la formation, les manuels, les associations...), celui des représentations et celui des pratiques ${ }^{7}$. Dans ce cadre, la notion de conscience disciplinaire est venue outiller le questionnement sur les représentations des acteurs et leurs modes d'élaboration (j'y reviendrai en 3).

Le second réseau, étroitement articulé au premier, est orienté vers la connaissance des phénomènes que tend à désigner la notion proposée dans trois directions complémentaires. l'appréhension systématique des (re)constructions des disciplines et de leurs variations; les relations entre ces (re)constructions et les modes d'enseignement; les relations entre ces (re)constructions et les catégories d'élèves. Cette dernière question s'accompagnait en outre d'une hypothèse très générale selon laquelle l'inégale réussite des élèves pourrait être liée à des modalités différentes de la conscience disciplinaire dans la mesure où les contenus, leur enseignement, les apprentissages et leur sens se trouvent principalement structurés, au sein de l'école, par le découpage disciplinaire et qu'il importe en conséquence de saisir les principes de fonctionnement et le sens de chacun des cadres disciplinaires. Ce qui consiste à avancer, de manière plus abrupte sans doute, que les variations de conscience disciplinaire pourraient participer de l’inégale réussite des élèves.

\section{Éléments pour une opérationnalisation méthodologique}

La mise en œuvre de ce concept s'est effectuée au travers de décisions portant sur les techniques de 
recueil, les matières scolaires et les niveaux du cursus pris en compte ainsi que sur les relations établies avec les catégories d'élèves et / ou de pratiques.

Trois techniques de recueil de données ont été jusqu'à présent utilisées. les questionnaires (à destination des élèves et des enseignants), des entretiens (eux aussi soumis à des élèves ou des enseignants) et des productions sollicitées, avec dans certains cas (notamment Constant-Berthe, 2005) des croisements de ces techniques. Mais, de fait, la base la plus importante des données recueillies est issue de questionnaires, organisés autour de trois questions reproduites pour chacune des matières (au sens scolaire du terme). "Qu'as-tu appris cette année en...? », "Comment sais-tu que tu es en cours de...? », «Selon toi, à quoi sert...? ${ }^{8}$. Ces trois questions renvoient, selon nous, à trois dimensions constitutives de la conscience disciplinaire. les contenus, les finalités et le repérage de l'espace de l'enseignement et des apprentissages dédié à telle ou telle matière. Il convient cependant de noter que, dans les travaux les plus récents menés en master, d'autres questions sont venues enrichir ce questionnaire, soit en amont afin d'obtenir des réponses mettant en jeu des catégories non imposées par le chercheur (par exemple, "Quelles matières as-tu étudiées cette année à l'école? »), soit en aval, afin d'affiner certaines réponses ou d'obtenir des éclairages par d'autres voies, par exemple les associations (« Donne trois mots auxquels te fait penser le français / les mathématiques...»)

Les recherches ont porté soit sur une seule matière (jusqu'à présent essentiellement français, mathématiques, technologie, musique, arts plastiques), soit sur une comparaison entre deux matières (français / mathématiques), soit sur l'ensemble des matières auxquelles étaient confrontés les élèves et cela soit à un seul niveau du cursus ( $6^{\text {ème }}$ ou CM 2 , qui fournit la majeure partie de nos données actuellement disponibles), soit sur un continuum (du CE 1 au CM 1 , de la $6^{\text {ème }}$ à la $\left.3^{\text {ème }}\right)$ soit selon des « coupes » (CM $\left.2 / 3^{\text {ème }} / 1^{\text {ère }}\right)$.

Les données recueillies ont enfin été mises en relation avec des catégories d'élèves (notamment selon la C.S.P. ou le niveau scolaire, tel qu'il était indiqué par les enseignants ou reconstruit par le chercheur à partir des notes obtenues), avec des modes de travail pédagogique (notamment « classique »vs « Freinet »), ou avec des organisations pédagogiques différentes (par exemple avec intervenant externe ou non, avec entrée par la production ou non), avec des pratiques extrascolaires liées à la matière enseignée (essentiellement dans les domaines artistiques).

\section{Premiers résultats}

Je me contenterai ici de rendre compte très succinctement des principaux résultats dont nous disposons à l'heure actuelle au travers de deux axes. celui des disciplines elles-mêmes et celui des relations avec le cursus, les modes d'enseignement et les catégories d'élèves.

\section{Variations de la conscience disciplinaire selon les matières}

Il convient tout d'abord de remarquer - ce qui va dans le sens de nos constats empiriques initiaux - que les notions de matière ou de discipline sont encore très instables jusqu'en CM 2. Ainsi, lorsque la question initiale est ouverte ( Quelles matières as-tu travaillées à l'école cette année? »), la liste des matières proposées par les élèves comprend, à côté des matières « officielles », des sous-domaines (lecture, poésie...), voire même des notions, surtout en français, mathématiques, activités physiques et sportives ou sciences. Et, lorsque les questions portent précisément sur telle ou telle matière, on peut rencontrer des absences de réponses ou des confusions, certaines pouvant être tributaires de la proximité phono-graphique des noms (géométrie / géographie).

Les diverses dimensions soumises au questionnement ne sont pas également perçues. Ainsi, quelle que soit la discipline, les finalités sont nettement moins identifiées que les contenus. Même s'il est vrai que la question posée est loin d'être facile, cela ne peut que faire écho aux recherches actuelles concernant le sens des apprentissages et la difficulté pour nombre d'élèves à construire à ce que l'on sollicite d'eux à l'école.

Les disciplines sont reconnues de manière très variable, que ce soit globalement ou par dimension soumise à l'investigation. Certaines sont, en tout cas, majoritairement bien reconnues. par exemple, au CM 2, français, mathématiques sport et langue. Suit un second groupe, constitué par histoire, sciences et musique. La reconnaissance de la géographie et 
des arts plastiques est moins assurée. Elle est très faible pour ce qui concerne l'éducation civique. Ces tendances sont intéressantes mais elles doivent être relativisées par certaines variations associées aux formes des questions. Ainsi, lorsque la première question laisse aux apprenants le choix des matières ( $Q$ Quelles matières as-tu travaillées à l'école cette année? »), certaines d'entre elles sont alors moins fréquemment mentionnées. C'est, par exemple, le cas du sport. On voit en tout cas que, sans recouper absolument le poids institutionnel des disciplines à l'école, l'identification des matières par les élèves n'est cependant pas sans entretenir des relations notables avec cette dimension.

Complémentairement, certaines matières sont plus ou moins «clivantes", leur reconnaissance engendrant une dispersion, voire une polarisation, plus ou moins forte. Ainsi, mathématiques ou sport, sont des disciplines moins clivantes que la géographie, par exemple.

Selon les cas, certaines dimensions ou certaines composantes des dimensions peuvent aussi, en raison de leur récurrence, apparaître comme des emblèmes, des marqueurs disciplinaires. Tel est le cas, par exemple, des frises en histoire, des mots " techniques », des cartes ou des plans en géographie, des nombres en mathématiques ou des dictées en français.

Les contenus des disciplines sont reconstruits au travers de principes d'organisation différents, avec quelques modalités principales. par sous-domaines, surtout en français (orthographe, grammaire, conjugaison, vocabulaire...) et en mathématiques (opérations, numération, mesures, géométrie...), par composantes « libres» (thèmes, notions...) par exemple en sciences ou par composantes « combinées ». périodes - personnages - événements en histoire. De surcroît, selon les cas, l'accent est plutôt porté sur les activités (géographie, sciences, arts, sports...) ou sur les savoirs.

Les objectifs et finalités des disciplines sont, comme je l'ai signalé précédemment, moins bien perçus que les contenus. non réponses, réponses quasi-tautologiques « [le français] ça sert à apprendre le français », réponses vagues centrées sur l'école («à réussir à l'école ») ou la vie extrascolaire ( «à avoir un métier plus tard »). Cependant, toujours au CM 2, certaines tendances apparaissent. finalités extrasco- laires importantes pour les langues, utilité dans la vie quotidienne pour la géographie, sport associé au développement personnel...

Le mode de repérage des disciplines est, lui aussi, très variable (toujours au CM 2) avec, dans certains cas, une entrée plus marquée par les contenus (mathématiques, histoire, sciences), dans d'autres, par des indicateurs matériels (géographie, arts, sports). Ces indicateurs sont plutôt mixtes dans le cas du français. Ils sont très rares en ce qui concerne l'éducation civique. Du côté des indicateurs «matériels », le discours du maître est très fréquemment cité. Les cahiers et les livres le sont en français. Les documents sont mentionnés de manière importante en sciences, histoire et géographie (avec en outre, dans ce dernier cas, cartes et globes). Le changement de salle constitue un repère récurrent en sport ou en musique. Enfin, il existe des indicateurs langagiers non négligeables, non seulement en langues vivantes mais encore, au travers des formulations des élèves, en mathématiques (chiffres et signes).

On peut encore remarquer, notamment au travers de la comparaison de descriptions en français et en mathématiques (Constant-Berthe, 2005), qu'il existe une différenciation disciplinaire dans les manières d'écrire cette catégorie de textes. Ainsi, ces écrits en mathématiques sont plus "neutres ", plus proches de définitions ou d'explications, plus courts, avec des phrases plus brèves, une moindre diversification dans les verbes choisis et les temps employés, plus de nombres écrits sous forme de chiffres, plus d'ajouts et de ratures et respectent moins les normes orthographiques et syntaxiques. Ils singularisent moins l'objet décrit, présenté sous un jour plus commun ou plus général qu'en français. Ces traits peuvent ainsi être considérés comme des traces possibles d'une conscience disciplinaire différenciée... sans que la différenciation soit l'objet d'un enseignement (au travers des déclarations des maîtres ${ }^{9}$ et des élèves).

Enfin, du côté des maîtres comme du côté des élèves, c'est surtout l'idée d'une étanchéité entre les matières qui semble dominer...

\section{Variations de la conscience disciplinaire selon élèves et enseignement}

Les études comparatives selon l'avancée dans le cursus (ainsi que la comparaison des études initia- 
lement non comparatives) mettent au jour d'importantes variations dans l'identification des disciplines et le rapport à celles-ci. contenus et enjeux mieux perçus, repérage plus assuré, mention d'un plus grand nombre d'exercices, rapport (positif ou négatif) plus marqué... On pourrait donc parler d'une véritable acculturation disciplinaire avec, dans certains cas, des résistances accrues à cette acculturation.

On a pu aussi construire des corrélations nettes entre conscience disciplinaire et niveau scolaire (bon / moyen / faible) des élèves. Les « bons » identifient plus de contenus (en accentuant plus les savoirs, sous forme de titres de leçons, que les activités); ils perçoivent plus d'enjeux, énoncés selon des modalités plus précises (notamment en ce qui concerne les enjeux extrascolaires); ils citent plus de disciplines lorsque la question inaugurale est ouverte... Ceci conforterait, au moins de manière tendancielle notre hypothèse sur les relations entre conscience disciplinaire et échec ou réussite scolaire (mais j'y reviendrai ultérieurement).

Nous avons encore pu établir des corrélations entre conscience disciplinaire et catégories socioculturelles des familles auxquelles appartiennent les élèves. Globalement, les élèves issus de milieux favorisés identifient mieux et détaillent plus les contenus que les autres (mais les écarts sont réduits en français, mathématiques, sport), construisent plus les disciplines au travers des titres des leçons (et moins au travers des sous-domaines et des activités) et perçoivent plus d'enjeux qu'ils formulent de manière plus précise. Il me semble que cela n'est pas sans générer des interrogations quant aux modalités familiales, ou plus largement extrascolaires, de construction de la conscience disciplinaire (j'y reviendrai en 3.4.).

Nous avons enfin pu mettre au jour qu'il existait des variations non négligeables dans les formes de conscience disciplinaire selon les modes de travail pédagogique (au CM 2). Ainsi, comparés à des élèves travaillant de manière classique, ceux qui sont soumis à la pédagogie «Freinet " ${ }^{10}$ présentent certaines singularités.

- ils mentionnent moins les disciplines "officielles » et citent plus d'activités spécifiques de ce mode de travail (recherches mathématiques, exposés, fiches, conférences, correspondance, entretiens...);

- ils énoncent moins de contenus sous forme de liste mais répondent plus longuement en décrivant telle ou telle activité, ce qu'ils ont pu y apprendre et si cela leur a plu ou non;

- ils indiquent plus d'enjeux dans des disciplines telles que français, mathématiques, géographie, sciences ou arts;

- ils citent plus fréquemment que les autres la lecture et / ou l'écriture en français et sont centrés, en mathématiques, sur les recherches mathématiques ${ }^{11}$;

- ils accordent, en matière de repérage, une plus grande importance aux activités et aux dispositifs.

Cela me parait appeler deux remarques. En premier lieu, cela tend à confirmer ce que nombre de constats, précédemment évoqués, rendaient visibles, à savoir que les formes de conscience disciplinaire sont fortement tributaires de l'enseignement et des configurations disciplinaires instaurées ${ }^{12}$. En second lieu, cela me semble inciter à prendre en compte comme composante de la conscience disciplinaire le rapport qu'établit l'élève avec la discipline concernée (j'y reviendrai en 3.4.).

\section{De quelques problèmes}

Il est clair - et les éléments de présentation n'auront pas manqué d'attirer l'attention là-dessus - que l'introduction possible de ce concept au sein de l'appareillage théorique des didactiques soulève de multiples problèmes. Je ne retiendrai ici, à titre provisoire, que quatre d'entre eux, parmi les plus importants à mes yeux.

\section{Questions de désignation et de sens ${ }^{13}$}

La désignation elle-même pose problème. Je ne l'ai d'ailleurs retenue qu'après de longues hésitations entre, par exemple, représentation de la discipline (terme présent dans le titre de mon article de 2003), image de la discipline (Colomb, 1993), conception de la discipline...

Outre les connotations liées au terme de conscience en raison de ses usages dans d'autres sphères disciplinaires (linguistique, philosophie, psychologie...), cette expression soulève trois problèmes majeurs à mon sens. Le premier est celui de l'articulation entre deux approches des phénomènes que ce concept vise à outiller. la première, descriptive, est celle des formes de 
conscience, appréhendées au travers des composantes sélectionnées, de leurs relations et de leur structuration en une totalité; la seconde, descriptive et évaluative est celle de «niveaux » ou de « degrés » de clarté ou de pertinence de cette (re) construction.

Cela soulève par voie de conséquence un second problème, celui des connotations « méta -» du terme de conscience. Or, dans mon maniement de ce concept, il n'existe pas nécessairement de conscience disciplinaire « consciente " (au sens d'explicite, de réfléchie). Elle peut être absente, inconsciente, présente simplement dans l'exercice de certaines pratiques ${ }^{14} \ldots$ ou encore faiblement construite. J'envisage donc la conscience disciplinaire comme une catégorie susceptible d'être actualisée selon des états de conscience très divers. Mais, et c'est là le troisième problème qui m'arrêtera ici, ces états de conscience sont évaluables, non seulement au regard de leur degré de construction ou d'objectivation pour le sujet lui-même mais aussi au regard de leur pertinence, ce qui pose la question de la référence de cette pertinence qui peut varier selon qu'il s'agit de la discipline telle qu'elle est construite (ou proposée) par les chercheurs en didactique, par les prescriptions officielles, par les recommandations (des formateurs, des associations...), par les représentations du / des maître(s), par les pratiques mises en place... Cela mérite donc d'être précisé à chaque fois...

Si malgré ces difficultés, j’ai choisi - au moins provisoirement - de maintenir l'expression de conscience disciplinaire, c'est pour quatre raisons principales. En premier lieu, je trouvais intéressant, dans le contexte théorique actuel, d'introduire un élément de rupture avec les termes de représentations et de rapports à, utilisés sans précautions ni méthodologiques (les glissements entre déclarations et représentations et la « naturalisation » de ces dernières sont, par exemple, fréquents), ni théoriques (la reprise de notions issues d'autres champs théoriques s'effectue bien souvent sans retravail critique en didactique). Au moins, cette rupture est susceptible d'introduire des éléments d'interrogation et de réinsister sur l'idée de construction (et par le sujet, et par le chercheur). La seconde raison tient à la combinaison possible que me semble favoriser cette expression entre formes et degrés, ce qui me paraît moins évident avec d'autres notions. En troisième lieu, conscience disciplinaire " unifie », solidarise les deux termes qu'elle conjoint, manifestant un des modes d'existence de la discipline (parmi d'autres sans doute), et rappelant ainsi qu'une discipline n'existe pas indépendamment de ses espaces d'actualisation. La quatrième et dernière raison tient finalement à l'importance de plus en plus grande que j'ai été amené à accorder aux phénomènes référés. Dans cette perspective, la notion de conscience disciplinaire insiste sur le fait que le «sujet didactique » ne se constitue véritablement qu'en tant qu'il est conscient du cadre disciplinaire où il s'inscrit et qu'il construit ses pratiques et les évalue à l'aune de ce cadre. Reste que, pour moi, le débat autour de la dénomination demeure ouvert et indispensable.

\section{Conscience disciplinaire / discipline}

La seconde famille de problèmes a trait à la relation instaurée entre conscience et discipline, l'expression retenue tendant à naturaliser le second terme. Or, la notion de discipline, si elle est fondamentale pour les didactiques et les didacticiens, n'a rien d'évident et s'avère particulièrement complexe à saisir, comme j'ai essayé de le montrer à plusieurs reprises (Lahanier-Reuter et Reuter, 2007; Reuter 2004), à la suite de Chervel (1988, 1998, 2006), en réfléchissant notamment à la distinction qu'il propose entre matière scolaire et discipline et en avançant la notion de configuration disciplinaire pour désigner les modes d'actualisation des disciplines selon les espaces (de prescriptions, de recommandations, de pratiques, de représentations...), les niveaux, les filières, les pays, les époques... Dans ce cadre, loin de participer à la naturalisation de la notion de discipline, le concept de conscience disciplinaire a, au contraire, vocation à la questionner. en interrogeant de manière indirecte les pratiques mises en place (j'y reviendrai en 3.3), en interrogeant l'intégration des cadres disciplinaires au travers de leur construction, en interrogeant les différences de représentations selon les espaces et selon les acteurs...

Reste que se pose la question du « début» des enseignements en tant qu'enseignements disciplinaires et on sait, de ce point de vue, qu'un certain nombre de didacticiens situent cela au collège..., ce qui rendrait caduque la majeure partie de nos investigations et de nos résultats. Sans vouloir minimiser un débat légitime et complexe mais qui nécessiterait un développement excédant le cadre de cet article, je me contenterai d'indiquer ici que mes positions sont sensiblement différentes en fonction des quelques arguments suivants. 
- les disciplines existent à mon sens à l'école primaire, via l'organisation des contenus formatés par la formation des maîtres, les instructions officielles ${ }^{15}$, les références à des savoirs savants ou à des champs de pratiques sociales, les manuels, les cours, les exercices, les évaluations, les horaires, les supports (cahiers, classeurs, documents...), les outils, les changements de salle, voire les changements d'intervenant;

- plus on avance dans le cursus primaire, plus cette organisation devient prégnante au point qu'on pourrait avancer qu'une des fonctions principales de ce lieu scolaire est l'acculturation disciplinaire.

\section{Problèmes méthodologiques}

Une troisième catégorie de problèmes regroupe ceux soulevés par les modes d'investigation expérimentés jusqu'à présent. Ici encore, je n'en retiendrai que quelques-uns parmi ceux qui me paraissent les plus importants.

En premier lieu, nous nous sommes principalement appuyés sur des déclarations sollicitées, questionnaires ou entretiens essentiellement, au détriment donc des manières de dire ou de faire non sollicitées, dans l'« ordinaire » de la classe (ce sur quoi nous essayons maintenant d'avancer).

En second lieu, les modes de recueil adoptés soulèvent des problèmes épineux. J'illustrerai cela d'abord à partir des questionnaires, ensuite à partir des productions. En ce qui concerne les questionnaires, outre leur longueur si on s'attache à plusieurs disciplines (ce qui nous a conduit à une passation en plusieurs fois au CM 2), s'est posée par exemple la question de la désignation même des matières, variable selon les instructions officielles, les maîtres, les modes de travail pédagogique... En ce qui concerne les productions, lorsque nous avons, par exemple, voulu comparer la conscience disciplinaire en mathématiques et en français, s'est posé le problème de la consigne. Si la consigne retenue, « Décris un pavé. », présente comme intérêts une tâche scripturale commune (décrire) et un référent commun (un pavé), facilitant la comparaison, on conviendra cependant que ni la tâche, ni le référent n'ont le même statut au sein des disciplines prises en compte (Constant-Berthe, 2005, 2006).

En troisième lieu, mais c'est là sans doute un lieu aussi stratégique que difficile, dans toute recherche, les problèmes liés au mode de traitement des données recueillies sont multiples. Comment pondérer réponses justes et erronées à une même question? Comment catégoriser les enjeux proposés par les élèves? Faut-il traiter de manière spécifique - et si oui, comment? - les réponses identiques de certains élèves à des questions différentes (contenus, enjeux, repérages)? Si on calcule le nombre de termes appropriés pour la question «Qu'as-tu appris cette année en français? », faut-il compter un, deux, trois ou quatre termes pour une réponse du type «les conjugaisons, le présent, le passé, le futur » ? On conviendra ici qu'il serait nécessaire d'expliciter et de discuter ces macro - et ces micro - décisions de traitement (ces dernières étant tout aussi importantes que les premières mais bien trop souvent occultées dans les écrits de recherche) afin de mieux comprendre comment les choix effectués formatent la production des résultats et quels seraient les plus pertinents.

\section{Les relations établies}

Le dernier type de problèmes sur lequel je m'arrêterai ici, en sachant qu'il aurait pu tout aussi bien trouver sa place dans le point précédent, concerne la pertinence didactique des pôles mis en relation.

De ce point de vue, il faut bien avouer que la relation entre conscience disciplinaire et niveau scolaire est insatisfaisante dans la mesure où risquent ainsi d'être confondus, sans précaution ni vérification, évaluation scolaire / professorale, elle-même obtenue de manière assez frustre (le classement en trois catégories d'élèves par les professeurs eux-mêmes ou par regroupement des notes en catégories par le chercheur), et évaluation par le chercheur lui-même des apprentissages disciplinaires qu'il estime cruciaux... ce qui soulève deux nouveaux problèmes non moins épineux. celui de la lourdeur de l'opérationnalisation nécessaire et celui de la conception disciplinaire du chercheur. Néanmoins, cela me paraît être, en l'occurrence, un problème à traiter de manière impérative.

\section{La conscience disciplinaire. intérêts en Didactique et en Didactique Comparée}

Sans sous-évaluer les problèmes soulevés par ce concept et son opérationnalisation méthodologique, il me semble néanmoins qu'il présente un certain nombre d'intérêts non négligeables, ce dont d'ailleurs les premiers résultats obtenus attestent, au moins en partie. C'est donc à l'explicitation de ces béné- 
fices que je souhaite consacrer la dernière partie de cet article. Dans cette perspective, je me permettrai d'abord de repréciser le sens que j'attribue au projet de connaissance des didactiques et de la didactique comparée avant de spécifier les intérêts du concept de conscience disciplinaire autour de trois axes. celui de l'appareillage conceptuel des didactiques, celui de l'outillage méthodologique et celui de l'instrumentation heuristique.

\section{Le projet de connaissance des didactiques et de la didactique comparée}

J'ai proposé de définir les didactiques comme les disciplines de recherche qui analysent des contenus (savoirs, savoir-faire...) en tant qu'ils sont des objets d'enseignement et d'apprentissage, référés / repérables à des disciplines scolaires (Reuter, 2005 ; Reuter, dir. 2007a). Sans occulter les questions redoutables attachées à la définition des notions de discipline de recherche et de discipline scolaire ainsi qu'aux modalités de référence des contenus, cette définition permet de porter l'accent sur l'importance des contenus et des disciplines scolaires qui garantit à mes yeux, au moins en partie, l'identité des didactiques, leur autonomie par rapport à d'autres disciplines de recherche, mais aussi la spécificité de chacune d'entre elles.

Le projet de connaissance de la didactique comparée $^{16}$, quant à lui, repose selon moi sur deux principes fondamentaux.

- la comparaison des disciplines scolaires et / ou des didactiques elles-mêmes, portant aussi bien sur leurs dimensions historiques, institutionnelles, épistémologiques, méthodologiques... que sur leurs fonctionnements « courants »;

- la construction d'outils - conceptuels et méthodologiques - à orientation similaire (ce qui fait que les didactiques ont une base commune dans leur projet de connaissance) mais permettant d'affiner leurs spécificités (ce qui fait que chaque didactique a une identité propre).

Dans cette perspective, si la didactique comparée possède bien une dimension autonome - dont l'assise institutionnelle et épistémologique demeure cependant largement en débat ${ }^{17}$-, elle se justifie cependant, en majeure partie, par la contribution qu'elle est susceptible d'apporter au projet de connaissance de chacune des didactiques et à l'affinement de la réflexion épistémologique que mène chaque didactique sur elle-même.

C'est donc dans le cadre ainsi tracé, que je me propose d'explorer certains des intérêts du concept de conscience disciplinaire.

\section{Conscience disciplinaire et appareillage conceptuel des didactiques}

Il me semble que le concept de conscience disciplinaire peut contribuer à la construction de l'appareillage conceptuel des didactiques en tant que celuici participe fondamentalement de leur identité et de leur autonomie. Il établit ainsi fermement le prisme disciplinaire, ce qui ne semble pas négligeable dans la mesure où, dans nombre de recherches, il n'est pas rare de constater des focalisations sur l'infradisciplinaire (tel savoir, tel savoir-faire...) ou des glissements vers le pédagogique (par exemple, lorsqu'il est question de contrat).

Indissociablement, il pose le principe que la représentation d'ensemble de la discipline est fondamentale à prendre en compte lorsqu'on étudie le rapport des acteurs à tel objet ou à telle pratique (même si ce n'est sans doute pas la seule dimension qui entre en jeu et même si, en retour, la construction du rapport à tel objet ou telle pratique contribue à la construction de l'image globale de la discipline). Cela me paraît fondamental à un moment du développement des didactiques où la spécialisation, sans doute incontournable, des chercheurs mène à des recherches pointues sur tel ou tel objet (par exemple, en français, l'oral, la lecture, l'écriture, la grammaire... voire le récit ou le personnage), recherches dont la précision se paie souvent d'un relatif aveuglement à l'insertion de cet objet au sein de l'ensemble disciplinaire, via les prescriptions, les pratiques, les représentations des enseignants ou des élèves... En d'autres termes, la recontextualisation ou la solidarisation des contenus, souvent évoquée lorsqu'on parle, par exemple, des mécanismes de la transposition didactique en général, me paraît souvent négligée lors des recherches singulières...

Enfin, et ceci me paraît particulièrement important, le concept de conscience disciplinaire permet de remodeler les emprunts à des notions précédemment évoquées (1.2.), telles que représentations, rapports à ou clarté, dans une perspective spécifi- 
quement didactique et cela au travers d'une orientation fondamentale, énonçable selon deux modalités complémentaires.

- en les mettant en relation avec le filtre disciplinaire qu'elles ignoraient tendanciellement - en fonction de leur socle d'origine - pour privilégier des objets infradisciplinaires (le personnage, la digestion...) ou transdisciplinaires (les savoirs, le langage...), en tout cas envisagés indépendamment de leur actualisation disciplinaire;

- en envisageant qu'en matière didactique, la (re) construction des objets et la construction d'une relation à ceux-ci ne se comprend qu'en tant qu'elle est tributaire d'interactions disciplinairement construites entre des sujets et des objets disciplinaires, ce qui constitue un garde-fou appréciable contre les figements naturalisants soit des sujets (qui seraient invariables quel que soit l'espace social où ils évoluent et sont appréhendés), soit des objets (qui seraient identiques quelles que soient les institutions et les pratiques qui les constituent), soit encore des rapports entre sujets et objets (eux-mêmes essentialisés).

Je soulignerai ici le fait qu'à mon sens, cette orientation est la condition sine qua non de la comparaison inter-disciplinaire.

\section{Conscience disciplinaire et outillage méthodologique}

Il me semble encore qu'ainsi conçu, le concept de conscience disciplinaire peut contribuer utilement à « outiller » méthodologiquement didactiques et didactique comparée en organisant la comparaison des disciplines scolaires autour de trois axes.

Le premier axe, le plus évident, est celui de leurs représentations et des variations de ces représentations, précédemment évoquées ( $c f .2$ ). formes des reconstructions, plus ou moins grande clarté dans l'appréhension des contenus, des enjeux, des repères de l'exercice disciplinaire, variations selon les catégories d'élèves... De ce point de vue, les premiers résultats obtenus me paraissent prometteurs.

Le second axe est celui des variations disciplinaires des relations entre les modalités de la conscience disciplinaire et les autres espaces qui construisent une discipline scolaire, particulièrement l'espace des prescriptions, l'espace des recomman- dations et l'espace des pratiques, que ces relations soient envisagées sous l'angle du poids de ces espaces sur la constitution de la conscience disciplinaire ou sous l'angle du poids des modalités de la conscience disciplinaire sur les autres espaces. dans la rédaction de nouveaux programmes ménageant des compromis avec l'existant, dans la confection des manuels scolaires, dans la mise en ouvre des pratiques par les enseignants ou par les élèves... Malgré des travaux qui vont dans ce dernier sens (même s'ils ont pu être menés à l'aide d'autres concepts, tels représentations ou contrat didactique), il me semble que ce second axe demeure encore, en grande partie, en friche.

Le troisième axe, qui participe au moins en partie du second, est celui de l'appréhension des pratiques. Dans cette perspective, les investigations menées à partir de et portant sur la conscience disciplinaire peuvent constituer un moyen, indirect, de reconstituer les pratiques. Doublement indirect sans doute dans la mesure où il ne s'agit ni d'observations, ni de déclarations centrées sur les pratiques. Mais néanmoins intéressant dans la mesure où la convergence d'un certain nombre de déclarations - elles-mêmes congruentes avec d'autres données issues d'autres modalités de recueil - amène à préciser certaines formes dominantes d'actualisation disciplinaire. par exemple, en ce qui concerne le français, la place importante accordée aux activités centrées sur la langue (orthographe, grammaire, conjugaison, vocabulaire...) et la place plus réduite allouée à l'oral ou à l'écriture.

\section{Conscience disciplinaire et instrumentation heuristique}

Il me semble encore que le concept de conscience disciplinaire présente des intérêts heuristiques non négligeables, excédant les questions initiales qui ont fondé son introduction. Afin d'étayer cela, je présenterai ici trois des pistes d'investigation que les résultats obtenus permettent d'ouvrir ou de préciser.

\section{Le paradoxe des modes de repérage}

$\mathrm{Au}$ vu des données obtenues lors du traitement des premiers questionnaires en réponse à la question. «A quoi vois-tu que tu fais (telle matière)? », nous avions construit quatre catégories dans les modes de repérage. 
- mode de repérage fondé sur les contenus. savoirs, savoir-faire...

- mode de repérage fondé sur les dimensions matérielles. supports, outils, emploi du temps, indications du maître, dispositifs, changement de salle, changement d'intervenant...

- mode de repérage mixte;

- mode de repérage inexistant.

Notre hypothèse, très générale, était que les élèves les meilleurs et / ou issus des milieux les plus favorisés se baseraient plus sur les contenus que les autres. Or, c'est l'inverse qui est apparu. ils se fondent plus sur la dimension matérielle.

À partir de là, nous avons envisagé plusieurs interprétations possibles. la plus grande confiance qu'auraient ces élèves dans le guidage qui leur est fourni; à l'inverse la complexité même du système proposé, notamment en ce qui concerne les supports (Chartier et Renard, 2000; Giguère et Reuter, 2003), qui nécessite un apprentissage loin d'être négligeable... Mais, en affinant les données obtenues, il nous semble qu'en fait il ne s'agit pas des mêmes indices matériels et, qu'à la différence des autres élèves, les élèves les meilleurs et / ou de milieux plus favorisés, s'appuieraient surtout des indicateurs liés à l'ordre scriptural (cahiers, classeurs, manuels...). Comme on le voit, si cela se confirme, ces résultats seraient de nature à étayer l'importance des relations entre forme scolaire et ordre scriptural (Vincent, dir., 1994; Lahire, 1993) et l'hypothèse d'une différenciation des performances des élèves en rapport avec la nature scripturale de la forme scolaire.

\section{Modes d'enseignement et conscience disciplinaire}

Une autre question que soulèvent les premiers résultats obtenus concerne les effets (i.e. ce que nous reconstruisons comme effets) des modes d'enseignement. Je n'en prendrai ici que deux exemples.

Le premier concerne les reconstructions des élèves travaillant selon le mode de travail pédagogique Freinet (cf. Reuter et Carra, 2005, et Reuter, dir. 2007 b). Elles se caractérisent en effet par un caractère moins " académique » au regard des textes officiels et des traditions scolaires en découpant ce qui s'effectue à l'école en référence à des activités centrales et typiques de ce mode de travail (les recherches mathématiques, les entretiens, la correspondance, les exposés...) tout en détaillant plus précisément ces activités, ce qui s'y est appris et la relation à cellesci. La question qui se pose alors, me semble-t-il, est de savoir si ce mode de reconstruction s'avère aussi pertinent pour faciliter les apprentissages et, ultérieurement, pour la poursuite du cursus disciplinaire au collège.

Le second exemple a trait à l'éducation civique tendanciellement d'autant moins identifiée qu'une partie de ses composantes (la vie de la classe, la citoyenneté scolaire...) ne fait pas l'objet d'un enseignement formel classique mais est incarnée sous formes de pratiques à vivre (conseils, débats...). Si, d'un côté, la conscience disciplinaire (quant aux contenus, aux enjeux, au repérage...) apparaît particulièrement faible, d'un autre côté, dans certains cas au moins, il semble bien que ces dispositifs participent de l'évolution souhaitée des normes et des pratiques des élèves. De nouveau, se pose donc la question des relations entre conscience disciplinaire et apprentissages ou, du moins, de leurs variations selon disciplines voire « quasi-disciplines » et contenus visés.

\section{Les sources de construction de la conscience disciplinaire}

Une autre piste de recherche ouverte par les premiers résultats obtenus est celle des sources de construction de la conscience disciplinaire, principalement chez les élèves.

Si la voie scolaire - intradisciplinaire semble fondamentale au vu des relations que nous avons pu effectuer avec les modes d'enseignement, les outils, les supports..., celle-ci ne me parait néanmoins pas exclusive si on considère notamment la différenciation selon les catégories socio-professionnelles dont relèvent les élèves d'une part, si on met ces données en relation avec toutes celles qui concernent l'éducation familiale et la réussite ou l'échec scolaire, d'autre part.

Deux directions sont donc empruntées à l'heure actuelle dans les recherches menées au sein de notre laboratoire. l'exploration des relations possibles avec les pratiques extrascolaires des élèves et l'exploration des relations possibles avec la conscience disciplinaire des familles.

On voit bien ici que ces directions soulèvent deux problèmes complexes. celui de l'opérationnalisation méthodologique et celui des frontières avec 
d'autres disciplines de recherche, telles la sociologie ou l'ethnologie.

Mais, dans le même mouvement, cela permet de comprendre en quoi les didactiques peuvent être des disciplines contributoires pour les autres en questionnant par exemple la sociologie sur l'absence de données qu'elle fournit sur cette dimension dont on peut cependant supposer qu'elle nourrit de manière non négligeable les échanges familiaux autour de la vie scolaire et dont on peut supputer l'importance dans la (re)production de la réussite ou de l'échec.

Cela permet en tout cas de confirmer, à mon sens, l'intérêt du concept de conscience disciplinaire comme instrument heuristique non seulement au sein des didactiques mais encore au sein des dialogues nécessaires entre didactiques et autres disciplines de recherche.

J'ai bien conscience (sans jeu de mots), au moment de conclure cet article, du caractère insatisfaisant de son propos. Sur bien des points, méthodologiques notamment, les problèmes ont plus été évoqués que véritablement traités. Je pense néanmoins qu'il peut présenter, pourvu que la lecture soit coopérative, un intérêt à deux niveaux. Le premier est celui qui a organisé explicitement cette contri- bution. la présentation et la discussion du concept de conscience disciplinaire, dont je pense avoir fondé, au moins en partie, les intérêts en didactiques et en didactique comparée. Il reste aux recherches et aux débats à venir à confirmer ou à infirmer ces intérêts. Cela me paraît faire partie des fonctionnements « ordinaires» des disciplines de recherche. Le second intérêt, plus rare me semble-t-il dans les fonctionnements évoqués, tient à l'espace accordé à cette exposition, dans ses diverses dimensions (définition, questionnements, genèse, intérêts, limites...), susceptible de permettre une véritable réflexion sur la fabrique (possible) de concepts (éventuels). Cette exposition, d'habitude implicitée ou disséminée en divers lieux théoriques, me parait intéressante, voire indispensable, aussi bien pour ceux qui ont participé à cette élaboration que pour ceux qui en sont les destinataires, non seulement pour mieux comprendre et évaluer telle ou telle proposition mais encore pour mieux réfléchir aux formes du travail théorique et à ses spécificités selon les domaines disciplinaires. En fin de compte, et quoi qu'il advienne des propositions que j'ai pu avancer, c'est tout autant à ce second niveau qu'au premier que j'espère avoir été un tant soit peu utile. 


\section{NOTES}

1. Voir aussi l'article «Conscience disciplinaire » dans le Dictionnaire des concepts fondamentaux des didactiques (Reuter, dir., 2007a).

2. Les principaux sont répertoriés dans la bibliographie de cet article. Beun, 2006; Clément, 2005; Kus, 2006; Lécuyer, 2005; Marhem, 2006; Roussel, 2006; Ségismont, 2004 et 2006; Van Meenen, 2004 et 2006. Voir aussi la thèse de Constant-Berthe (2005).

3. Voir, par exemple, l'étude de Chambon (1990).

4. Encore que certains travaux, par exemple l'étude de Lebeaume (2000) sur découverte du monde, sciences et technologie aux cycles II et III soit fort proche de notre problématique.

5. Voir aussi, dans cette perspective, les recherches plus récentes que nous avons menées sur le récit (Pratiques, 2007).

6. Je simplifie ces thèses, en réduisant notamment le caractère interactif des relations entre clarté quant à l'objet et apprentissages techniques.

7. Voir notamment sur la notion de discipline scolaire, Lahanier-Reuter et Reuter 2007, et Reuter, 2004.

8. Ce double choix (questionnaire, trois questions de base) est bien sûr fort discutable (j'y reviendrai en 2.3). Il a cependant permis de dresser assez rapidement un arrière-plan quantitativement non négligeable et de préciser certaines pistes de questionnement (cf. 3.4).

9. Qui sont d'ailleurs étonnés lorsqu'une telle suggestion leur est faite lors de l'entretien...

10. Voir, sur ce point, outre le mémoire de Van Meenen (2006), la présentation de nos recherches dans Reuter et Carra, 2005 et Reuter dir. 2007b.

11. Pour une présentation de cette activité très spécifique, voir notamment Lahanier-Reuter, 2005.

12. Cela n'est certes pas bouleversant de nouveauté mais, nécessitait d'être confirmé en tenant compte des variations selon les disciplines.

13. Je tiens ici à remercier François Jacquet-Francillon pour l'éclairage critique dont il m’a fait bénéficier.

14. On pourrait rapprocher cela de ce que désignent certains termes ou expressions usités dans d'autre domaines, tels « épilinguistique » ou « logique de l'action » Avec un problème méthodologique à ne pas sousestimer. le questionnement suscite chez l'interviewé une posture réflexive ou, tout au moins, une attention accrue aux phénomènes soumis à l'investigation.

15. Quelles que soient leurs variantes de surface... auxquelles résistent les pratiques.

16. Voir, pour une mise en place fondamentale de ce projet, le numéro 141, de la Revue Française de Pédagogie, coordonné par A. Mercier, M.L. Schubauer-Léoni et G. Sensevy.

17. Y compris quant aux formes de travail de son exercice (collectives ou liées à une qualification qui reste à définir).

\section{BIBLIOGRAPHIE}

Beun, M. (2006). Variations des représentations des élèves de collège sur la discipline technologie, Mémoire de master 1, Université Charles de Gaulle - Lille 3.

Brossard, M. (1994). L'adaptation de l'enfant à l'école, Paris : Stablon. 
Brossard, M. (2002). Des maîtres, des élèves et des tâches, in Dolz, J., Schneuwly, B., Thévenaz-Christen, T., Wirthner, M., (Eds). Les tâches et leurs entours en classe de français, C.D. Rom des Actes du $8^{\text {e }}$ congrès international de la D.F.L.M., Neuchâtel, septembre 2001 (diffusion A.I.R.D.F.).

Cahiers pédagogiques, (1999). Décrire dans toutes les disciplines, n³73, Paris, CRAP, avril.

Chambon, M. (1990). Les représentations des disciplines scolaires par les parents d'élèves. enjeux de valeurs, enjeux sociaux, Revue française de pédagogie, n 92, 31-40.

Chartier, A.M., Renaud P. (2000). Cahiers et classeurs. supports ordinaires de travail scolaire, dans Plane S. et Schneuwly, B., dir, Repères, nº 22, Les outils d'enseignement du français, Paris, INRP, 135-159.

Chervel, A. (1988). Lhistoire des disciplines scolaires. réflexion sur un domaine de recherche, Histoire de l'éducation, $\mathrm{n}^{\circ}$ 38, Paris, INRP, mai, 59-119.

Chervel, A. (1998). La culture scolaire. Une approche historique, Paris, Belin.

Chervel, A. (2006). Histoire de l'enseignement du français du XVII ${ }^{e}$ au XX $X^{e}$ siècle, Paris, Retz.

Clément, M. (2005). La conscience disciplinaire des élèves de sixième. Étude comparée dans deux collèges de milieux socio-culturels différents, Mémoire de master 2, Sciences de l'Éducation, Université Charles de Gaulle - Lille 3.

Colomb, J., dir. (1987). Les enseignements en CM 2 et en sixième. Ruptures et continuités, Paris, INRP.

Colomb, J. (1993). Contrat didactique et contrat disciplinaire, dans Houssaye J., dir., La pédagogie. une encyclopédie pour aujourd'hui, Paris, E.S.F., 39-50.

Constant-Berthe, N. (2005). Décrire en français et en mathématiques. Étude comparée de représentations et de pratiques. Thèse, Université Charles de Gaulle - Lille 3, 8 décembre.

Constant-Berthe, N. (2006). Pourquoi et comment élaborer une consigne identique et pertinente dans deux disciplines différentes (mathématiques et français) ? In Perrin-Glorian M.J. et Reuter Y., dir. (2006). Les méthodes de recherche en didactique, Villeneuve d'Ascq, Presses Universitaires du Septentrion, 71-81.

Delcambre, I., Reuter, Y. (2002). Images du scripteur et rapports à l'écriture, Pratiques, nº 113-114, Images du scripteur et rapports à l'écriture, Metz, CRESEF, 7-28.

Downing, J., Fijalkow, J. (1984). Lire et raisonner, Toulouse, Privat.

Enjeux (2000), La description, ${ }^{\circ} 47$ / 48, Namur, CEDOCEF, mars /juin.

Giguère, J., Reuter Y. (2003). Les cahiers et classeurs et la construction de l'image de la discipline à l'école primaire, Actes du colloque international de Bordeaux, 3-5 avril. Construction des connaissances et langage dans les disciplines d'enseignement (C.D. Rom).

Jaubert, M. (2001). Cohérence textuelle et positionnement énonciatif contextuellement pertinent en classe de sciences, in Bernié, J.-P., dir. (2001). Apprentissage, développement et significations, Pessac, Presses Universitaires de Bordeaux.

Kus, A. (2006). Les élèves de CM 2 et leur conscience disciplinaire en Arts Plastiques, Mémoire de master 1, Université Charles de Gaulle - Lille 3.

Lahanier-Reuter, D. (2005). Enseignement et apprentissages mathématiques dans une école Freinet, Revue Française de Pédagogie, nº 153, Décrire, analyser, évaluer les pédagogies nouvelles, Paris, INRP, 55-65.

Lahanier-Reuter, D. (2006). La statistique. une discipline scolaire? Communication aux $32^{\mathrm{e}}$ journées de statistique, Société Française de Statistique, Clamart, 29 mai- 2 juin.

Lahanier-Reuter, D., Reuter, Y. (2002). Écrits et apprentissages. Première approche dans quatre disciplines au collège, Pratiques, n 113-114, Images du scripteur et rapports à l'écriture, juin, 113-134.

Lahanier-Reuter, D., Reuter, Y. (2007). Lanalyse de la discipline. quelques problèmes pour la recherche en didactique, dans Falardeau, E., Fisher, C., Simard, C., Sorin, N., dir. La didactique du français. Les voies actuelles de la recherche, Quebec, Presses de l'Université Laval, 27-42. 
Lahire, B. (1993). Culture écrite et inégalités scolaires. Sociologie de "l'échec scolaire "à l'école primaire, Lyon, Presses Universitaires de Lyon.

Lautier, N. (1997). À la rencontre de l'histoire, Villeneuve d'Ascq, Presses Universitaire du Septentrion.

Lebeaume, J. (2000). Jeux d'étiquette, jeux de kim, jeux de famille, puzzles ou devinettes à l'école. Découverte du monde, sciences et technologie aux cycles II et III, Aster, n 31, Les sciences de 2 à 10 ans, Paris, INRP, $197-215$.

Lecuyer, C. (2005). La conscience disciplinaire des élèves. Les élèves décrivent-ils différemment en français et en technologie?, Mémoire de master 1, Université Charles de Gaulle - Lille 3.

Marhem, A. (2006). La conscience disciplinaire en éducation musicale chez les élèves de CM 2, Mémoire de master 1, Université Charles de Gaulle - Lille 3.

Mercier, A., Schubauer-Léoni, M.L., Sensevy G., eds (2002). Revue Française de Pédagogie, nº 141, Vers une didactique comparée, Paris, INRP, octobre-novembre-décembre.

Pratiques (1998), La description, n 99, Metz, CRESEF, septembre.

Pratiques (2007), Récits et disciplines scolaires, n 133-134, Metz, CRESEF, juin.

Reuter Y. , dir. (1998). La description. Théories, recherches, formation, enseignement, Villeneuve d'Ascq, Presses Universitaires du Septentrion.

Reuter, Y. (2000). La description. Des théories à l'enseignement-apprentissage, Paris, E.S.F.

Reuter, Y. (2003). La représentation de la discipline ou la conscience disciplinaire, La Lettre de la DFLM, $\mathrm{n}^{\circ} 32$, Namur, diffusion AIRDF, 18-22.

Reuter, Y. (2004). Analyser la discipline. quelques propositions, La Lettre de l'AIRDF, n 35, Namur, 5-12.

Reuter, Y. (2005). Didactique du français. éléments de réflexion et de proposition, dans Chis, J.-L., David, J., Reuter, Y., dir.. Didactique du français. Fondements d'une discipline, Bruxelles, De Boeck, 211-234.

Reuter, Y., dir. (2007a). Dictionnaire des concepts fondamentaux des didactiques, Bruxelles, De Boeck.

Reuter, Y., dir. (2007b). Une école Freinet. Fonctionnements et effets d’une pédagogie alternative en milieu populaire, Paris, l'Harmattan.

Reuter, Y., Carra, C. (2005). Analyser un mode de travail pédagogique « alternatif ». L'exemple d'un groupe scolaire travaillant en pédagogie "Freinet », Revue Française de Pédagogie, n 153, Décrire, analyser, évaluer les pédagogies nouvelles, Paris, INRP, octobre-novembre-décembre, 39-53.

Roussel, A. (2006). La conscience disciplinaire en français des collégiens, Mémoire de master 2, Université Charles de Gaulle - Lille 3.

Ségismont, C. (2004). La conscience disciplinaire des élèves de CM 2. Étude auprès d’une population de milieu peu favorisé, Mémoire de maîtrise, Université Charles de Gaulle - Lille 3.

Ségismont, C. (2006). La conscience disciplinaire des élèves de CM 2. Étude complémentaire, Mémoire de master 2, Université Charles de Gaulle - Lille 3.

Van Meenen, E. (2004). La conscience disciplinaire des élèves de CM 2. Étude auprès d’une population de milieu favorisé, Mémoire de maîtrise, Université Charles de Gaulle - Lille 3.

Van Meenen, E. (2006). La conscience disciplinaire des élèves de CM. 2. Étude auprès de deux classes aux modes de fonctionnement pédagogiques différents, Mémoire de master 2, Université Charles de Gaulle - Lille 3.

Vincent, G., dir. (1994). Léducation prisonnière de la forme scolaire? Scolarisation et socialisation dans les sociétés industrielles, Lyon, Presses Universitaires de Lyon. 